
las Ciencias

ISSN: 1697-011X

revista.eureka@uca.es

Universidad de Cádiz

España

\title{
¿Qué saben niños y niñas sobre evolución? Diseño y aplicación de un modelo científico escolar de evolución para educación primaria
}

\author{
Vázquez-Ben, Lucía; Bugallo-Rodríguez, Ánxela \\ ¿Qué saben niños y niñas sobre evolución? Diseño y aplicación de un modelo científico escolar de evolución \\ para educación primaria \\ Revista Eureka sobre Enseñanza y Divulgación de las Ciencias, vol. 19, núm. 1, 2022 \\ Universidad de Cádiz, España \\ Disponible en: https://www.redalyc.org/articulo.oa?id=92068491007 \\ DOI: https://doi.org/10.25267/Rev_Eureka_ensen_divulg_cienc.2022.v19.i1.1102
}


Fundamentos y líneas de trabajo

\title{
¿Qué saben niños y niñas sobre evolución? Diseño y aplicación de un modelo científico escolar de evolución para educación primaria
}

\begin{abstract}
What do primary school children know about evolution? Design and application of a school scientific model of evolution for primary education
\end{abstract}

Lucia Vázquez-Ben

Departamento de Pedagoxia e Didáctica, Facultade de

Ciencias da Educación, Universidade da Coruña, España

lucia.vben@udc.es

iD https://orcid.org/0000-0003-1685-5671

Anxela Bugallo-Rodríguez

Departamento de Pedagoxía e Didáctica, Facultade de

Ciencias da Educación, Universidade da Coruña, España

anxela.bugallo@udc.es

iD https://orcid.org/0000-0002-8059-7083
DOI: https://doi.org/10.25267/

Rev_Eureka_ensen_divulg_cienc.2022.v19.i1.1102

Redalyc: https://www.redalyc.org/articulo.oa?

$\mathrm{id}=92068491007$

Recepción: 20 Abril 2021

Revisado: 13 Septiembre 2021

Aprobación: 15 Septiembre 2021

\section{Resumen:}

En este artículo se presenta un modelo científico escolar diseñado como instrumento para la enseñanza de la teoría evolutiva en educación primaria. Ha sido elaborado a partir de una selección de ideas nucleares y niveles escalares que fueron organizados en forma de heptágono. Con este formato el modelo podría incrementar su complejidad en etapas educativas posteriores. Para comprobar su utilidad, se ha usado en la creación y análisis de una prueba de evaluación inicial sobre evolución, aplicada a un grupo de estudiantes de $5^{\circ}$ de educación primaria. En sus respuestas muestran un gran número y variedad de preconcepciones relacionadas con las ideas nucleares pero, a diferencia de lo que ocurre en secundaria, son escasas las que puedan representar un obstáculo. Estos resultados reafirman la necesidad de comenzar la enseñanza de esta teoría desde etapas tempranas y de una forma progresiva.

PALABRAS CLAVE: modelo escolar, evolución, preconcepciones, educación primaria.

\section{ABSTRACT:}

This paper presents a school scientific model designed to serve as an instrument for teaching the theory of evolution in primary education. The model was developed from a selection of evolutionary core ideas and biological levels of scale later organised into a heptagon. This format could allow to gradually increase its complexity in later stages. To check its utility, this model has been employed to create and analyse an initial assessment on evolution aimed to a class-group of fifth graders (10-11 years old). Findings show students at this age have numerous and diverse preconceptions related to evolutionary core ideas. However, very few of those preconceptions could be considered an obstacle to their learning; just the opposite to what usually happens in secondary school. Such an outcome reinforces the convenience of introducing this content from early stages and in a progressive way.

KEYWORDS: school models, evolution, preconceptions, primary education.

\section{INTRODUCCIÓN}

Los modelos son herramientas que emplean los científicos y científicas para representar (mental o materialmente) algún aspecto del mundo, así que su forma y extensión están condicionados por su objetivo último (Giere 2004). Permiten describir un fenómeno natural, explicarlo, hacer predicciones sobre el mismo, o comunicar sus ideas a otras personas (Oh y Oh 2011). Son, a la vez, producto e instrumento de la actividad 
científica (Gilbert 1993), lo que los convierte en su núcleo principal (Giere 1999), razón por la cual deberían ser determinantes en el proceso de enseñanza.

La defensa de su uso como elemento estructurador del curriculum de ciencias (Gilbert y Justi 2016; Martí 2012) conlleva cambios en la visión del proceso de enseñanza, pues cada disciplina se vertebraría en torno a sus modelos principales, a partir de los cuales se irían desarrollando el resto de los contenidos. En esta línea, García Rovira (2005) prescinde de hablar de teorías o conceptos científicos y, en su lugar, opta por organizarlo en torno a unos pocos, pero significativos modelos teóricos; concretamente para el ámbito de la Biología, establece cuatro modelos básicos: el de célula, el de ser vivo, el de ecosistema y el de evolución, que engloba a todos los anteriores. Este último modelo no está presente en la normativa española de educación primaria, aunque sí está incluido en los curricula de esa etapa en otros países de referencia como Estados Unidos, Reino Unido, Francia o Suecia (Vázquez-Ben y Bugallo-Rodríguez 2018).

Aprender esta teoría progresivamente, y desde edades tempranas, ayudaría a evitar que las experiencias que niños y niñas tienen antes de su enseñanza formal lleguen a ser tan determinantes, que impidan a los estudiantes alcanzar una comprensión profunda y adecuada del fenómeno evolutivo en etapas posteriores (Hermann 2011).

Para su tratamiento adecuado en primaria, los docentes de este nivel educativo precisan, y solicitan, una mayor formación sobre esta temática y contar con los recursos que les permitan trabajarla en el aula (Asghar, Wiles y Alters 2007; Vázquez-Ben y Bugallo-Rodríguez 2017). Esta reclamación implica la necesidad de aportar modelos de evolución que orienten el trabajo de los docentes, y que logren integrar todos los conocimientos que deben construirlo. Estamos hablando de proponer modelos científicos escolares que reflejen la relación entre los modelos básicos englobados dentro de la teoría, que puedan usarse en diversos pasos del proceso de enseñanza y que ayuden al profesorado a la creación de los suyos propios.

\section{MARCO TEÓRICO}

\section{Los modelos científicos escolares}

Los modelos científicos escolares (Adúriz-Bravo e Izquierdo 2009) son representaciones didácticas de los modelos científicos, que tienen como fin la progresión de los modelos mentales del alumnado. Son el resultado de la transposición didáctica (Oliva, Aragón, Bonat y Mateo 2003), adaptando los saberes científicos a las características del alumnado, tales como su estado de desarrollo evolutivo y sus especificidades personales y socioculturales, haciéndolos así más asequibles (Chamizo 2010).

Como instrumentos de enseñanza, su función no es sustituir ni erradicar los modelos mentales de los estudiantes, sino facilitar su progresión. De este modo, el aprendizaje se convierte en una evolución sucesiva de los modelos mentales que ya posee el alumnado, utilizando como referente el modelo científico escolar que se presenta desde la escuela (Clement 2000) y que delimita cuál es el conocimiento deseable.

Para conseguir este objetivo, el modelo científico escolar puede tomar diversas formas en el aula (Gilbert y Justi 2016), cada una con una demanda conceptual diferente (Harrison y Treagust 2000). Será tarea del docente evaluarlos detenidamente y seleccionar el más adecuado en cada caso.

El profesorado es, pues, el encargado de seleccionar o elaborar ese modelo científico escolar, y de utilizarlo como eje para organizar y secuenciar el aprendizaje del alumnado (Gilbert y Justi 2016). En su diseño hay que tener en cuenta, en primer lugar, cómo su uso en el proceso de enseñanza-aprendizaje va a condicionar la transposición desde el modelo científico original, y aceptar su progresividad, pues es un modelo que debe incrementar gradualmente su complejidad y abrirse a la interacción con otros modelos. En segundo lugar, los docentes deben tener claro que el objetivo del proceso de instrucción es la evolución desde el modelo mental 
creado por el propio alumnado previamente, a partir de sus propias concepciones (Passmore, Svobod y Giere 2014), no la imposición del modelo científico escolar.

Son los modelos mentales de los estudiantes los que constituyen el verdadero instrumento de aprendizaje, lo que significa que aprender ciencias no implica ninguna habilidad especial, sino simplemente tomar conciencia y aplicar habilidades que, como seres humanos, ya poseemos y empleamos de forma natural a diario (Vosniadou 2013). El conocimiento de las preconcepciones que el estudiante presenta puede ayudar a acercarnos a su modelo mental previo, a través del modelo expresado, que es la verbalización o representación del modelo mental que posee un determinado sujeto (Gilbert 2004).

\section{La teoría evolutiva como familia de modelos}

Las teorías, constituidas por enunciados y sus representaciones, se consideran una familia de modelos (Giere 1988). En el caso de la evolución, su aprendizaje requiere de la integración de los tres modelos subyacentes (célula, ser vivo y ecosistema) y que el alumnado sea capaz de: (1) coordinar el razonamiento a nivel de organismo (ser vivo) y población(es), con el razonamiento sobre distribuciones y cambios en dichas distribuciones; (2) así como, evaluar simultáneamente cuáles puedan ser los mecanismos, en sus diferentes escalas (celular, individuo y población), que provocan dichos cambios (Lehrer y Schauble 2012).

Esta compleja y, a la vez, clave teoría científica no puede tratarse como un tema más del curriculum de Biología (McVaugh, Birchfield, Lucero y Petrosino 2011), ni relegarse al final de la escolaridad obligatoria, tal y como ocurre en la educación española con las grandes teorías científicas. Como indican Criado, CruzGuzmán, García-Carmona y Cañal (2014, p. 2) «la introducción de las ideas científicas clave, que explican un amplio rango de fenómenos, no se puede hacer directa y abruptamente porque resultarían abstractas y sin significado».

Otra de las consecuencias del tardío tratamiento es que, para entonces, el alumnado ha ido confeccionando numerosas y muy diversas concepciones que pueden entrar en conflicto con el modelo científico escolar, y que se han ido reforzando con el paso del tiempo (Roldán Muñoz 2008). Lo muestra la amplia literatura acerca de las dificultades que experimenta el alumnado de secundaria para comprender este contenido (p.ej. Banet y Ayuso 2003; Gregory 2009).

Las referencias constantes al fenómeno evolutivo en la vida cotidiana, derivadas del uso del lenguaje, del contacto con narraciones mágico-religiosas, enseñadas y aprendidas en entornos familiares, sociales y escolares (Alters y Nelson 2002), unidas a la propia naturaleza del pensamiento infantil (eminentemente vitalista, esencialista, antropocéntrico y teleológico; Martí 2012), conducen a la formación de concepciones sobre este tema desde muy temprana edad (Hermann 2011).

Para evitar que estas concepciones acaben siendo un obstáculo sería necesario trabajar progresivamente este modelo científico desde la educación infantil y primaria. De hecho, las grandes teorías científicas, como la evolución, deberían convertirse en eje vertebrador del curriculum en las diferentes etapas educativas. Como familias de modelos que son, ayudarían a que el objetivo del proceso de enseñanza fuera la creación de esa red integradora del resto de los modelos básicos de cada disciplina, que se irían conectando entre sí y originando nuevos resultados fruto de la globalidad de la visión dada por la teoría.

\section{La construcción de un modelo científico escolar de evolución}

Para comprender el mundo a través de esta teoría, se debería ir «sembrando el pensamiento evolutivo en el alumnado» (Lehrer y Schauble 2012) ya desde educación infantil y primaria, trabajando diferentes ideas, dimensiones y niveles de organización, que también orientarán el diseño de los modelos científicos escolares. 
En las dos últimas décadas los estudios han presentado diferentes selecciones de ideas, conceptos o áreas de cara a la enseñanza del modelo de evolución. En la propuesta de progresión de Catley, realizada junto a Lehrer y Reiser (2005), se citan las siguientes «grandes ideas» (bigideas): diversidad, relación entre estructura y función, ecologia/interrelaciones, variación, cambio y procesos geológicos. Defienden la necesidad de tratarla junto con prácticas de argumentación y herramientas matemáticas, que consideran esenciales para desarrollar el pensamiento evolutivo. Posteriormente, Lehrer junto a Schauble (2012) ha marcado tres constructos: variabilidad, cambio y ecosistema.

Andersson y Wallin (2006) incluyen, entre los aspectos específicos del contenido a tratar, el papel del azar, además de variación de rasgos heredables, adaptación y acumulación, tiempo evolutivo y selección natural ligada a las tasas de supervivencia y de reproducción.

McVaugh junto a otros autores (2011) hablan de cuatro áreas principales (core areas): variación, selección, herencia y tiempo evolutivo. Pobiner (2016) enuncia ocho core building concepts: variación, diversidad, competición, supervivencia, adaptación, mutación, herencia y tiempo evolutivo.

Afrontar la elaboración de un modelo científico escolar para una teoría, como es el caso de la evolución, significará ser capaz de integrar una selección de esas ideas nucleares y desarrollarlas progresivamente, en todos los niveles de organización afectados.

Estos niveles se corresponden a aquellos en los que operan y con los que establecen generalizaciones: los modelos biológicos básicos de célula, ser vivo y ecosistema (Gómez, Sanmartí y Pujol 2007). La necesidad de abordar los diferentes niveles escalares, y explicitar cómo interactúan entre sí, es una reclamación de los especialistas (Andersson y Wallin 2006; Catley et al. 2005; Lehrer y Schauble 2012) pero, al mismo tiempo, es una de las principales dificultades que encuentra el alumnado a la hora de comprender la evolución (Harms y Reiss 2019).

El modelo científico escolar ha de construirse respondiendo a esta necesidad de progresión e integración. Debe servir para orientar el avance de los modelos mentales del alumnado, siempre a partir del conocimiento de sus preconcepciones, que han determinado su modelo previo. Partir de ellas es fundamental para organizar el aprendizaje del modelo de evolución.

Estas afirmaciones han suscitado las preguntas de investigación del presente trabajo:

- ¿Cómo elaborar un modelo científico escolar destinado a la enseñanza de la teoría evolutiva en Educación Primaria?

- ¿Ese modelo científico escolar puede utilizarse para analizar las respuestas del alumnado en una prueba de evaluación inicial?

- ¿Las preconcepciones que presenta el alumnado en este nivel educativo sobre las ideas nucleares seleccionadas pueden ser un obstáculo para su avance?

\section{Metodología}

Este artículo se enmarca en un proyecto de investigación más amplio, que tiene como principal objetivo el diseño, implantación y revisión de recursos para una construcción progresiva y efectiva del modelo de evolución biológica desde educación primaria. Con tal fin se ha diseñado un modelo científico escolar en desarrollo, partiendo de escoger las ideas principales y de buscar un modo de representación que integrara esas ideas y los modelos inmersos en la teoría evolutiva.

\section{La selección de las ideas nucleares}

La selección de las ideas nucleares necesarias para la construcción del modelo ha sido el resultado de utilizar cuatro vías complementarias: 
(1) La revisión bibliográfica de estudios que presentan una selección de conocimientos para el aprendizaje progresivo de la evolución biológica desde las primeras etapas.

(2) El estudio del curriculum de diferentes países realizado por Vázquez-Ben y Bugallo-Rodríguez (2018), donde se identifican las ideas sobre evolución incluidas en sus normativas para educación primaria.

(3) Una entrevista semiestructurada a especialistas en Didáctica de las Ciencias relacionados con la enseñanza de la evolución y la genética. Se entrevistaron individualmente un total de 12 especialistas de diferentes países y universidades (Argentina, España, Estados Unidos, Grecia, Israel, Suiza, Suecia). Por motivos de agenda y preferencia de las personas entrevistadas, cinco entrevistas se realizaron por correo electrónico; el resto fueron presenciales. En cuanto a la forma de registro, en el caso de las presenciales, dos fueron grabadas en audio (previo permiso del participante); en las otras cinco, la persona que entrevistaba (autor 1) fue tomando notas que más tarde se remitieron a los participantes para su revisión y corrección de ser el caso (validación del respondiente; Simons 2011). Entre las preguntas formuladas se incluyó «¿Qué conceptos habrían de trabajarse y qué metodología debería emplearse?».

(4) Un grupo de discusión integrado por tres docentes en ejercicio de primaria y secundaria, cuyas intervenciones fueron grabadas en audio (previo permiso de los participantes). Durante la sesión se abordó el tema de qué contenidos deberían trabajarse en educación primaria en relación con la evolución biológica.

Esta multiplicidad de vías ha permitido atender a las distintas perspectivas de la realidad estudiada (Stake 2010): la literatura, las normativas curriculares que marcan los contenidos, los docentes que tendrán que impartirlos (profesorado de educación primaria) o que ya lo hacen (profesorado de educación secundaria), así como los especialistas que se encargarán de la formación docente (inicial y continua), y de proporcionar los recursos y estrategias más adecuadas.

Los datos recogidos en las entrevistas y el grupo de discusión se sometieron de forma conjunta a un proceso de análisis cualitativo compuesto de tres fases (Miles, Huberman y Saldaña 2013): 1) reducción de datos, donde una vez identificadas las unidades de análisis, siguiendo criterios espaciales y temáticos (Rodríguez, Gil y García 1996), se procedió a su codificación inicial y focalizada; 2) disposición y transformación de los datos en una misma matriz, facilitando así su comparación (entre sí y con respecto a los resultados obtenidos a partir de la revisión bibliográfica y el análisis curricular); y 3) obtención y verificación de conclusiones, que dio lugar a la selección definitiva de las ideas nucleares.

\section{Diseño y uso del modelo científico escolar propuesto}

En cuanto al diseño, esto es, la forma y formato del modelo científico escolar, se ha tomado como punto de partida el modelo de modelización propuesto por Gilbert y Justi (2016). El modelo de estos autores refleja claramente cómo interactúan entre sí las distintas fases, un aspecto especialmente significativo en este caso, pues la construcción del modelo de evolución precisa de una visión integrada de las distintas ideas. Sin embargo, el modelo en desarrollo se ha adaptado a dos dimensiones, para facilitar su utilización por parte del profesorado en aula a través de diagramas y otros materiales.

Para recoger los niveles escalares (celular, de organismo y de población) se ha usado la estructura del modelo de modelos biológicos básicos presentado por García Rovira (2005).

Este modelo científico escolar en desarrollo ha servido para el diseño y aplicación de una propuesta didáctica sobre evolución en un aula de $5^{\circ}$ educación primaria. Tanto los estudiantes como la docente participante, era la primera vez que trabajaban sobre evolución. La muestra está formada por 19 estudiantes, de entre 10 y 11 años, cuyos nombres empleados en este estudio son ficticios.

La propuesta se inicia con una prueba de evaluación inicial (Anexo 1) compuesta por cinco problemas que requieren utilizar las ideas nucleares en diferentes niveles de organización escalar. En este artículo se presenta el diseño y análisis de resultados de esta prueba inicial, así como la aplicación del modelo científico escolar propuesto para evaluar los modelos expresados por el alumnado. Como el modelo expresado es el que 
corresponde al dominio público, mientras que el material es personal y privado (Gilbert y Boulter, 1998), al estudiar los primeros no estamos analizando los modelos mentales de los estudiantes. Los modelos expresados son limitados a la hora de conocer el modelo mental de los sujetos y están condicionados por las creencias de cada individuo (Franco y Colinvaux 2000).

Dado que esa expresión será diferente según la forma de representación elegida, tal y como indican Boulter y Buckley (2000) quienes distinguen los modelos expresados según el tipo de actividades llevadas a cabo, en esta prueba se han incluido cuestiones que promueven respuestas de dos tipos: verbales (descripción, argumento, explicación) y visuales (dibujos).

Las respuestas del alumnado fueron sometidas a un doble proceso de análisis. Primero, se hizo uso del software MAXQDA 2018 para realizar su codificación inicial ( $1^{\text {a }}$ fase) y focalizada (2a fase) (Miles $e t$ al. 2013), lo que permitió catalogar las principales preconcepciones presentadas por los estudiantes. Luego, tomando como referencia el modelo científico escolar elaborado, se procedió a identificar qué ideas nucleares empleaba cada estudiante en sus respuestas, así como en qué niveles de organización escalar operaba, para construir la representación de sus modelos iniciales.

\section{Resultados y Discusión}

\section{Una propuesta de modelo científico escolar de evolución}

Fruto del análisis conjunto de la revisión bibliográfica, la comparativa curricular, la entrevista a especialistas y el grupo de discusión (Tabla 1), el modelo científico escolar sobre evolución para educación primaria que se propone en este trabajo se compone de siete ideas nucleares: biodiversidad, variabilidad, selección natural, adaptación, herencia, ancestros comunes y cambio. Como se puede ver en la Tabla 1 se han remarcado esas siete ideas (subrayado), de forma que se observa su representatividad en los diversos ámbitos, especialmente en el curricular que es el usado como referencia, pues recoge las propuestas de aquellos países donde se está impartiendo la teoría evolutiva en esta etapa educativa. Para cada idea se han distinguido tres niveles de organización escalar: célula (que incluye el molecular), individuo y población.

Es importante señalar que, de las siete ideas nucleares seleccionadas, en el curriculum español de primaria solo se incluyen dos: biodiversidad, que se recoge en la Orden Ministerial ECI/3960/2007, y adaptación, que solo aparece en las normativas de nueve comunidades autónomas. 
TABLA 1

Ideas nucleares propuestas desde los distintos ámbitos: literatura, curriculum y profesionales

\begin{tabular}{|c|c|c|c|c|c|c|c|}
\hline \multicolumn{5}{|c|}{ Literatura } & \multirow[b]{2}{*}{$\begin{array}{c}\text { Análisis } \\
\text { curricular } \\
\text { (Vázquez-Ben } \\
\text { y Bugallo- } \\
\text { Rodríguez } \\
\text { 2018) } \\
\text { ideas nucleares }\end{array}$} & \multicolumn{2}{|c|}{ Profesionales } \\
\hline $\begin{array}{l}\text { Catley et } \\
\text { al. (2005) } \\
\text { big ideas }\end{array}$ & \begin{tabular}{|c|} 
Andersson y \\
Wallin \\
(2006) \\
content specific \\
aspects
\end{tabular} & $\begin{array}{c}\text { McVaugh } \\
\text { et al. } \\
\text { (2011) } \\
\text { core ideas }\end{array}$ & $\begin{array}{l}\text { Lehrer y } \\
\text { Schauble } \\
\text { (2012) } \\
\text { building } \\
\text { blocks }\end{array}$ & $\begin{array}{l}\text { Pobiner } \\
\text { (2016) } \\
\text { core building } \\
\text { concepts }\end{array}$ & & $\begin{array}{c}\text { Entrevista a } \\
\text { especia- } \\
\text { listas en } \\
\text { Didáctica }\end{array}$ & $\begin{array}{c}\text { Grupo } \\
\text { discusión } \\
\text { docentes } \\
\text { primaria y } \\
\text { secundaria }\end{array}$ \\
\hline $\begin{array}{l}\frac{\text { Variación }}{\text { Diversidad }} \\
\text { Cambio } \\
\text { Ecología/ } \\
\text { Interrela- } \\
\text { ciones } \\
\text { Estructura- } \\
\text { función } \\
\text { Procesos } \\
\text { geológicos }\end{array}$ & $\begin{array}{l}\frac{\text { Variación de }}{\text { rasgos }} \\
\underline{\text { heredables }} \\
\underline{\text { Adaptación }} \\
\text { Acumulación } \\
\underline{\text { Selección }} \\
\frac{\text { natural }}{\text { Tiempo }} \\
\text { evolutivo } \\
\text { Rol del azar }\end{array}$ & $\begin{array}{l}\frac{\text { Variación }}{\text { Selección }} \\
\frac{\text { Herencia }}{\text { Tiempo }} \\
\text { evolutivo }\end{array}$ & $\frac{\text { Variabilidad }}{\text { Cambio }}$ & $\begin{array}{l}\frac{\frac{\text { Variación }}{\text { Adaptación }}}{\text { Diversidad }} \\
\frac{\text { Herencia }}{\text { Tiempo }} \\
\text { evolutivo } \\
\text { Mutación } \\
\text { Competición } \\
\text { Supervivencia }\end{array}$ & $\begin{array}{l}\frac{\text { Variación de }}{\text { rasgos }} \\
\frac{\text { Adaptación }}{\text { Biodiversidad }} \\
\frac{\text { Cambios }}{\text { (organismo y }} \\
\frac{\text { población) }}{\text { Herencia de }} \\
\frac{\text { rasgos }}{\text { heredados vs }} \\
\frac{\text { adquiridos) }}{\text { Selección }} \\
\frac{\text { natural }}{\text { Evidencia de }} \\
\frac{\text { ancestros }}{\text { comunes }} \\
\text { Selección } \\
\text { artificial } \\
\text { Extinción }\end{array}$ & $\begin{array}{l}\frac{\text { Variabilidad }}{\text { intraespecífi- }} \\
\frac{\text { ca }}{\text { Adaptación }} \\
\frac{\text { Biodiversi- }}{\frac{\text { dad }}{\text { Cambio }}} \\
\frac{\text { Herencia }}{\text { Selección }} \\
\underline{\text { natural }} \\
\text { Existencia de } \\
\text { fósiles } \\
\text { Mutación } \\
\text { Selección } \\
\text { artificial } \\
\text { Evolución } \\
\text { humana }\end{array}$ & $\begin{array}{l}\frac{\text { Adaptación }}{\text { Biodiversi- }} \\
\frac{\text { dad }}{\text { Cambio }} \\
\frac{\text { Ancestros }}{\text { comunes }} \\
\text { Pruebas de } \\
\text { evolución } \\
\text { Evolución } \\
\text { humana }\end{array}$ \\
\hline
\end{tabular}

Para elegir la forma de representación de estas ideas en el modelo se ha partido de la figura propuesta por Gilbert y Justi (2016) anteriormente citada. Se ha buscado que el modelo fuera aprehensible, funcional y fácil de usar (Norman 1983), lo que ha llevado a probar varias posibilidades. Inicialmente se escogió un heptaedro, que fue descartado debido a la dificultad para su presentación $2 \mathrm{D}$ dado su número de unidades. La siguiente opción desarrollada fue una configuración hexagonal donde cada idea clave era representada por un hexágono regular; las seis figuras convergerían en un hexágono central (la evolución). El resultado fue un panal de celdas que posibilitaba reflejar el carácter dinámico y continuo que debía tener el modelo. Podían incluirse otras ideas clave o, incluso, subdividir cada uno de estos hexágonos, por ejemplo para incluir mutación y reproducción sexual en variabilidad.

Finalmente, la representación escogida es un heptágono regular (figura 1), que además de facilitar la incorporación de nuevas ideas al modelo, presenta otras ventajas respecto a las anteriores propuestas: 


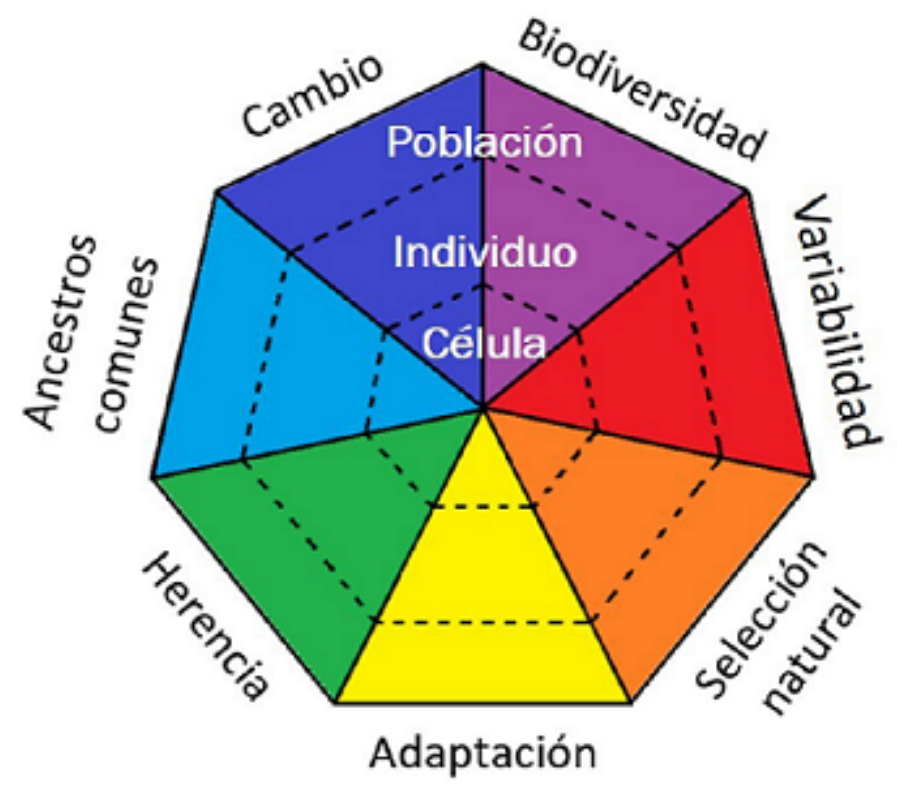

FIGURA 1

Modelo científico escolar de evolución biológica propuesto para educación primaria

1. Se distribuye en siete secciones iguales, reflejando así que todas y cada una de las ideas nucleares gozan de la misma importancia.

2. Al estar compuesto por siete triángulos iguales que comparten su vértice, se refuerza visualmente el carácter integrado del modelo; es decir, para lograr la construcción efectiva del modelo de evolución, habrán de desarrollarse las siete ideas en su conjunto, y a diferentes niveles de organización escalar (representados por las subdivisiones concéntricas de cada triángulo). Estos niveles de organización escalar no responden a niveles jerárquicos, sino que se trata de ser capaz de operar en cada uno de ellos y, sobre todo, identificar cómo interactúan entre sí.

3. Es una figura familiar, fácil de recordar y de representar en 2D. Asimismo, se ha asociado un color a cada idea nuclear y se ha marcado la existencia de interacción entre niveles escalares a través de líneas discontinuas para facilitar la interpretación de los resultados, en el caso de la investigación, o la propia evaluación del alumnado, en el caso de su aplicación a la práctica docente.

\section{La prueba de evaluación inicial}

Este modelo ha determinado el diseño de la prueba utilizada para conocer las concepciones del alumnado sobre las diversas ideas nucleares, así como su capacidad para operar en diferentes niveles escalares (Anexo 1):

Problema $\mathrm{n}^{\circ}$ 1: se entrega al alumnado una imagen del esqueleto de las extremidades anteriores de varios animales (uso de órganos homólogos), preguntándoles qué tienen en común y diferente (biodiversidad) y a qué se puede deber (ancestros comunes).

Problema $\mathrm{n}^{\mathrm{0} 2}$ : deben explicar en una familia de gatos compuesta por la pareja progenitora y su descendencia (herencia), por qué una de las crías en particular, a diferencia de sus hermanos/as y sus progenitores, habría nacido con el pelo largo (variabilidad).

Problema nº3: a través del caso de las rosas blancas teñidas de rojo y su posible descendencia (berencia y no cambio), que aparece en la novela Alicia en el Pais de las Maravillas, se comprueba si consideran la herencia de caracteres adquiridos, una concepción que puede resultar un obstáculo importante (Gregory 2009). 
Problema $\mathrm{n}^{\circ}$ : se centra en la diversidad de tonos de piel del ser humano (biodiversidad y adaptación). Este problema permite contrastar la noción de adquisición de rasgos (tonos más oscuros) con la de pérdida de rasgos (tonos más claros), que es mucho más difícil de adquirir que la primera, razón por la cual Ha y Nehm (2014) recomiendan trabajarla de forma específica, en relación con la de adquisición, para evitar que se asuma como simple "falta de necesidad" o "desuso".

Finalmente, el problema $n^{0} 5$ introduce la selección natural, recurriendo a un caso real: la diferencia de color y distribución de los ratones de Nuevo México, que posibilita operar con todas las ideas nucleares.

Las cuestiones planteadas son de carácter abierto y precisan de la aplicación de las ideas nucleares. Para reforzar la necesidad de buscar una justificación la docente les pidió de forma continua que explicaran sus respuestas («Explica por qué piensas eso»). Esto permite que sus respuestas sirvan para caracterizar mejor los modelos (expresados) previos del alumnado, tanto a nivel de conocimientos, como en su capacidad para representar, describir, explicar y argumentar.

Tras aplicar esta prueba y analizar las respuestas producidas por el alumnado, se ha podido constatar la presencia de un elevado número y variedad de preconcepciones en torno a la evolución y también a la genética (Tabla 2), pese a no haber recibido enseñanza formal al respecto. La mayoría son concepciones útiles para construirse una explicación del mundo natural e, incluso, las incoherentes para la ciencia son «plausibles» para su interpretación. Destaca también su empleo del vocabulario específico sobre esta temática, pues términos como evolución, genes, genética, ADN y mutación aparecen en sus respuestas.

En cuanto a las ideas movilizadas, es interesante el hecho de que varias veces hayan recurrido a ideas nucleares diferentes de las previstas inicialmente para cada tarea, utilizándolas en apoyo de su argumento. Por ejemplo, en el problema $n^{\circ} 4$ sobre la diversidad de tonos de piel, diseñado para usar las ideas de biodiversidad y adaptación, Zeltia recurrió además a la idea de herencia: «Por el ADN de nuestros padres o por la luz solar, por eso cuando vamos a la playa nos podemos morenos». 
TABLA 2

Ejemplos de respuestas escritas para cada problema e idea nucleares movilizadas por los estudiantes

\begin{tabular}{|c|c|c|c|}
\hline $\mathbf{N}^{\circ}$ & $\begin{array}{c}\text { Problema } \\
\text { (resumido) }\end{array}$ & Ejemplos de respuestas escritas & $\begin{array}{l}\text { Idea nuclear } \\
\text { movilizada }\end{array}$ \\
\hline \multirow[t]{2}{*}{1} & $\begin{array}{l}\text { a) ¿Qué tienen en } \\
\text { común y diferente el } \\
\text { ser humano, el perro, } \\
\text { un ave y una ballena? }\end{array}$ & $\begin{array}{l}\text { «Tienen en común: son seres vivos (animales), pueden } \\
\text { desplazarse de un sitio a otro, son vertebrados. Se } \\
\text { diferencian: sus huesos no están en la misma posición; } 1 \\
\text { nada, } 1 \text { vuela, } 2 \text { caminan; no se alimentan de lo mismo» }\end{array}$ & Biodiversidad \\
\hline & $\begin{array}{l}\text { b) ¿Por qué tienen un } \\
\text { esqueleto tan parecido } \\
\text { viviendo en ambientes } \\
\text { tan diferentes? } \\
\text { (imagen) }\end{array}$ & $\begin{array}{l}\text { «Porque hace mucho tiempo todos los animales mamíferos } \\
\text { eran algo parecido a una rata» }\end{array}$ & $\begin{array}{l}\text { Ancestros } \\
\text { comunes }\end{array}$ \\
\hline \multirow[t]{3}{*}{2} & \multirow{3}{*}{$\begin{array}{l}\text { Una pareja de gatos } \\
\text { con pelo corto ha } \\
\text { tenido una camada. } \\
\text { Todos los gatitos } \\
\text { tienen el pelo largo } \\
\text { excepto uno. ¿Cómo es } \\
\text { posible? }\end{array}$} & «En su familia pudieron tener un gato con pelo largo» & \multirow{2}{*}{$\begin{array}{l}\text { Herencia } \\
\text { Variabilidad }\end{array}$} \\
\hline & & $\begin{array}{l}\text { «Tal vez los gatos tuvieran distintos genes y si se fusiona dan } \\
\text { una raza de gato con pelo largo» }\end{array}$ & \\
\hline & & $\begin{array}{l}\text { «Porque a medida que crecen los gatitos van perdiendo el } \\
\text { pelo y algunos acaban con él corto como estos dos» }\end{array}$ & Cambio \\
\hline \multirow[t]{2}{*}{3} & \multirow{2}{*}{$\begin{array}{l}\text { Tras teñir las rosas } \\
\text { blancas de rojo, ¿el } \\
\text { próximo año saldrán } \\
\text { rojas o blancas? }\end{array}$} & "Que sí, que de unas rosas blancas no pueden salir rojas» & \multirow{2}{*}{$\begin{array}{l}\text { (No)Cambio } \\
\text { Herencia }\end{array}$} \\
\hline & & $\begin{array}{l}\text { «Opino que Cinco de Picas tiene razón, porque si las rosas en } \\
\text { principio son blancas, sus descendientes también» }\end{array}$ & \\
\hline \multirow[t]{4}{*}{4} & \multirow{4}{*}{$\begin{array}{l}\text { ¿Por qué crees que } \\
\text { tenemos la piel de } \\
\text { tantos colores } \\
\text { diferentes? }\end{array}$} & $\begin{array}{l}\text { «Por el lugar donde nacimos o descendemos, y por el clima } \\
\text { que hace en ese lugar» }\end{array}$ & \multirow[t]{2}{*}{$\begin{array}{l}\text { Adaptación } \\
\text { Biodiversidad }\end{array}$} \\
\hline & & $\begin{array}{l}\text { "Creo que es por el lugar típico donde vivimos; por ejemplo, } \\
\text { suele haber muchos negros en África porque allí hace mucho } \\
\text { calor y hace que la piel sea más oscura» }\end{array}$ & \\
\hline & & $\begin{array}{l}\text { «Porque cada uno nace con un color diferente; también a } \\
\text { veces de donde seas puedes tener la piel de muchos colores» }\end{array}$ & $\begin{array}{l}\text { Biodiversidad } \\
\text { Variabilidad }\end{array}$ \\
\hline & & $\langle$ Por nuestro origen, raza o $A D N »$ & Herencia \\
\hline \multirow[t]{10}{*}{5} & \multirow{6}{*}{$\begin{array}{l}\text { a) ¿Por qué hay ratones } \\
\text { claros y ratones } \\
\text { oscuros? }\end{array}$} & «Porque tiene más pelo uno que el otro» & Variabilidad \\
\hline & & $\begin{array}{l}\text { «Pues que unos viven más en la lava y son más oscuros y los } \\
\text { otros son más claros porque están más en el desierto» }\end{array}$ & $\begin{array}{l}\text { Variabilidad } \\
\text { Adaptación }\end{array}$ \\
\hline & & $\begin{array}{l}\text { «Dependiendo del lugar donde habitan estos ratones, el color } \\
\text { de su piel cambia para protegerse de los depredadores» }\end{array}$ & $\begin{array}{l}\text { Adaptación } \\
\text { Cambio }\end{array}$ \\
\hline & & $\begin{array}{l}\text { «Porque cada uno tiene el pelo de un color diferente (como } \\
\text { su madre o su padre). Es igual que nosotros» }\end{array}$ & \multirow[t]{2}{*}{$\begin{array}{l}\text { Variabilidad } \\
\text { Herencia }\end{array}$} \\
\hline & & $\begin{array}{l}\text { «A que a uno de los ratones le alcanzó el volcán, pero } \\
\text { sobrevivió; entonces sus hijos tuvieron el mismo pelo» }\end{array}$ & \\
\hline & & $\begin{array}{l}\text { «Por la erupción volcánica algunos ratones quedaron de color } \\
\text { oscuro y los demás blancos» }\end{array}$ & $\begin{array}{l}\text { Variabilidad } \\
\text { Cambio }\end{array}$ \\
\hline & \multirow{4}{*}{$\begin{array}{l}\text { b) ¿Por qué hoy en día } \\
\text { hay más ratones } \\
\text { oscuros que claros en } \\
\text { la zona de lava? }\end{array}$} & $\begin{array}{l}\text { «Porque puede ser que los ratones estuvieran adaptados a su } \\
\text { tierra y por eso tenían color claro antes de la erupción y } \\
\text { después de ella se adaptaron al color oscuro» }\end{array}$ & $\begin{array}{l}\text { Adaptación } \\
\text { Cambio }\end{array}$ \\
\hline & & $\begin{array}{l}\text { «Por la reacción química que expulsó el volcán, que hizo que } \\
\text { varios ratones sobrevivieran, pero con cambios» }\end{array}$ & Cambio \\
\hline & & $\begin{array}{l}\text { «También puede ser por mutaciones que produce al } \\
\text { desarrollarse con ratones de una especie diferente» }\end{array}$ & $\begin{array}{l}\text { Biodiversidad } \\
\text { Herencia } \\
\text { Cambio }\end{array}$ \\
\hline & & $\begin{array}{l}\text { «Puede que los ratones de color oscuro se fueron allí para } \\
\text { vivir mejor, ya que se pueden camuflar y los animales no los } \\
\text { ven» }\end{array}$ & $\begin{array}{l}\text { Adaptación } \\
\text { Variabilidad }\end{array}$ \\
\hline
\end{tabular}


Utilizando el modelo científico escolar elaborado se han representado los modelos iniciales del alumnado. Los distintos colores asignados marcan las secciones correspondientes a cada idea nuclear y nivel escalar abordados por el estudiante, tomando en cuenta tanto sus respuestas escritas como gráficas (dibujos). Por ejemplo, Tomás propone que la diferencia de distribución entre los ratones en Nuevo México es «porque puede que los ratones de color oscuro se fueron allí para vivir mejor, ya que se pueden camuflar y los animales no los ven», aludiendo así no al mecanismo de selección natural, pero sí a la migración y a la adaptación. Complementa su explicación escrita con el siguiente dibujo (figura 2), de especial interés pues muestra claramente la diferencia de frecuencia y distribución de ratones oscuros y claros en la población (variabilidad), cuando lo esperable en estas edades era que adjudicaran cada color a una zona, como sucedió con muchos otros de sus compañeros y compañeras.

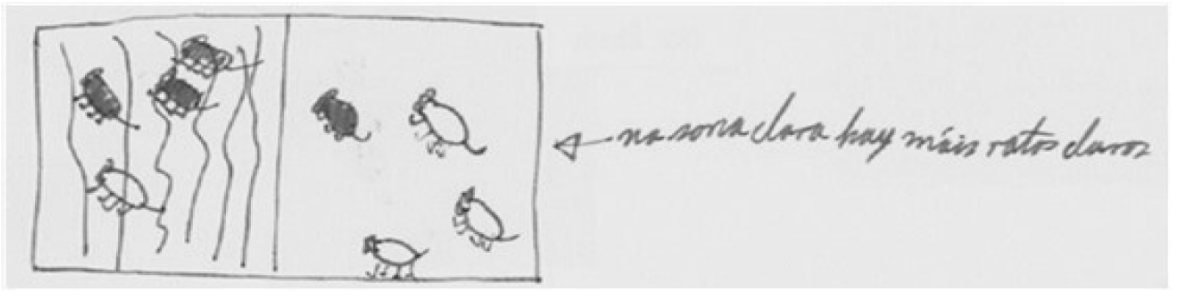

FIGURA 2

Dibujo de Tomás representando la diferencia de distribución de ratones claros y oscuros

En la figura 3 se puede apreciar con claridad cómo todos los estudiantes están familiarizados con al menos tres de las siete ideas nucleares definidas para el modelo científico escolar elaborado. De hecho, la mayoría de los participantes (16/19) recurre al menos a cuatro ideas nucleares: biodiversidad (morado), variabilidad (rojo), herencia (verde) y cambio (añil) o adaptación (amarillo), que son las más frecuentes.

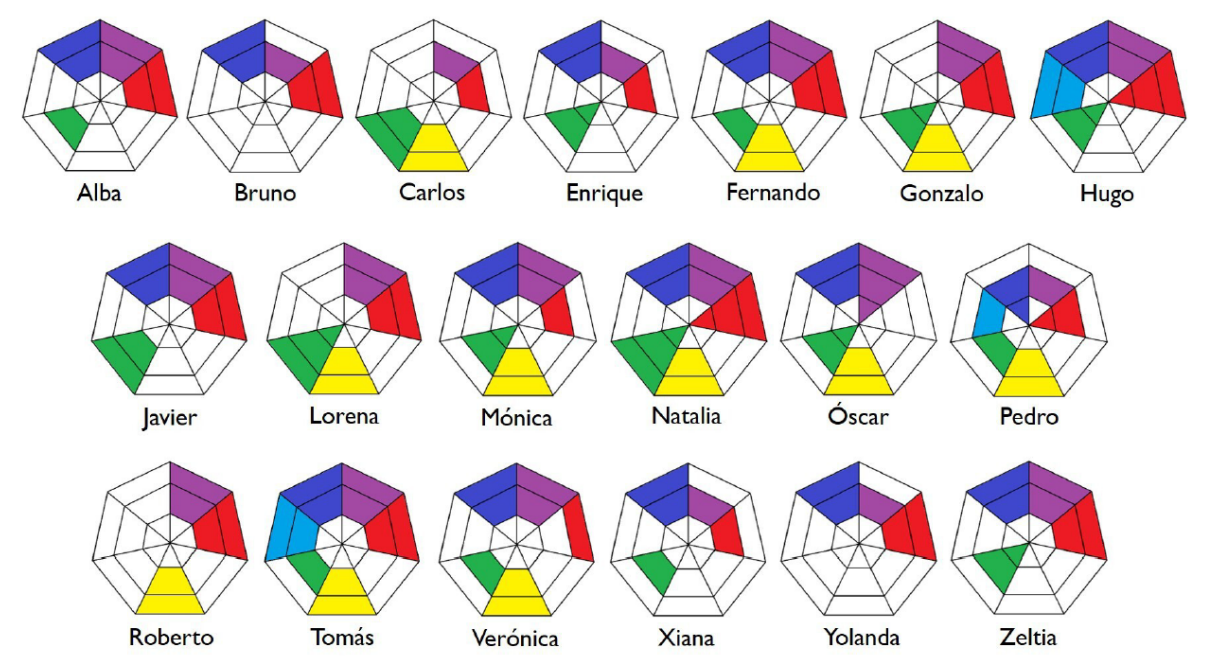

FIGURA 3

Reconstrucción de los modelos expresados iniciales del alumnado

En cambio, entre las ideas nucleares citadas, la menos habitual es ancestros comunes, que solo mencionan tres alumnos: Hugo, que hace alusión al hecho («Porque todos son "avances" de un animal que existió hace muchos milenios»); Pedro, que trata de explicar el mecanismo («todo el cuerpo de los animales ha ido evolucionando y a lo mejor se centra en un mismo espécimen y no lo alcanzaron, será como un humano a cuatro patas, o así, por eso sus huesos se parecen más al de los humanos»); y Tomás, que comenta que «hace mucho tiempo todos los animales mamíferos eran algo parecido a una rata», recurriendo a la clase de animal desde un punto de vista evolutivo y no meramente clasificatorio. Ningún estudiante hizo alusión explícita a 
la selección natural, aunque respuestas como la de Natalia («Por la reacción química que expulsó el volcán, que hizo que varios ratones sobrevivieran, pero con cambios») resultan de interés de cara a esta idea.

$\mathrm{Al}$ examinar las formas de razonamiento del alumnado, parecen ser capaces de construir explicaciones en cierta medida elaboradas, útiles, aun cuando no se correspondan con la visión científica. Por ejemplo, Bruno o Zeltia, que atribuyen el largo del pelo del gatito a un mayor tiempo de vida/desarrollo embrionario, o Yolanda, que lo relaciona con cambios corporales derivados del crecimiento de la especie. Sin embargo, su capacidad de justificar y argumentar varía en función de:

- El/la estudiante: algunos se limitan a contestar de manera descriptiva, sin llegar a justificar su respuesta; por ejemplo, Xiana determina que las rosas «seguirán dando rosas blancas. Pero si quisieran rojas tendrían que cambiarlas», pero no explica cómo ni por qué.

- El problema tratado: por ejemplo, Mónica justifica todas sus respuestas e, incluso, recurre a los datos proporcionados en el problema $5 \mathrm{~b}$ ( $\ll$ En la zona de desierto hay muchos animales que comen ratones entonces muchos escaparon para la zona de la lava»), pero no lo hace así en la pregunta dedicada a homologías $\left(\mathrm{n}^{\circ} \mathrm{b}\right.$ ), que ha sido también la menos respondida (solo 12 de 19 estudiantes). Es posible que el enunciado de esta segunda pregunta del problema $\mathrm{n}^{\circ} 1$ haya condicionado y limitado sus respuestas, al centrarse en la semejanza entre las extremidades a nivel óseo y al representarlas aisladamente.

En cuanto a la presencia de preconcepciones que pudieran suponer un obstáculo en la comprensión de este contenido, lo cierto es que se han detectado muy pocas, y las que fueron así consideradas han de matizarse. Por ejemplo, solo uno de los estudiantes recurrió al creacionismo, que usó para explicar el parecido entre especies: «Porque todos fueron creados en la tierra». Sin embargo, estrictamente hablando, no hace referencia a ninguna entidad sobrenatural, por lo que tampoco se podría asegurar que se refiera a un origen incompatible con la perspectiva científica.

Igualmente, solo otro aludió a la evolución como progreso, y el hecho de que coloque entre comillas la palabra "avances" («Porque todos son "avances" de un animal que existió hace muchos milenios», Hugo) haría pensar más en una forma de expresarse que en una afirmación de progreso.

Se descarta, en este nivel educativo, considerar como un problema las alusiones a la adaptación por necesidad, hechas por cinco estudiantes, ya que esta idea constituye uno de los pasos intermedios desde el pensamiento teleológico hacia la selección natural. La noción de adaptación basada en las necesidades podría convertirse en un problema grave a largo plazo si se consolida el pensamiento teleológico, pero en estas edades si se usa como punto de partida puede servir como un trampolín para el pensamiento evolutivo (Legare, Lane y Evans 2013). La diferencia radica en abordar estas ideas antes de que se conviertan en un obstáculo (casi) insuperable.

Respecto a la idea de herencia de caracteres adquiridos, que, recordemos, se buscaba específicamente en el problema de las rosas, todos los estudiantes concluyeron que las rosas de la siguiente generación serían blancas, a pesar de haberlas teñido de rojo. Sin embargo, en el problema de los ratones la mitad de los estudiantes asociaron la diferencia de color de los ratones a la erupción volcánica; cuatro incluso lo atribuyeron a haberse «manchado» con la ceniza: «Porque por la ceniza volcánica los ratones de la zona se tiñeron de color oscuro» (Javier).

Estos resultados sugieren que esta concepción podría activarse solo en el caso de causas naturales, pero no artificiales, lo que implicaría un empleo selectivo, y, por tanto, consciente, de este razonamiento. Por otra parte, con el descubrimiento de la epigenética, sería interesante cuestionar hasta qué punto esta idea puede ser un obstáculo.

Lo mismo sucede en los casos de generación de nuevos caracteres por combinación de dos individuos o especies diferentes que citan dos estudiantes. En sus respuestas no emplean el nivel de organismo, como podría esperarse, sino el molecular, y, por lo tanto, compatible con el conocimiento actual sobre genes acumulativos: «Tal vez los gatos tuvieran distintos genes y si se fusiona dan una raza de gato con pelo 
largo» (problema n², Hugo); y «También puede ser por mutaciones que produce al desarrollarse con otros ratones de una especie diferente» (problema $\mathrm{n}^{\circ} 5$, Natalia).

A este respecto, es posible que el número y orden de presentación de los datos en el problema $n^{\circ} 5$ de los ratones de Nuevo México haya contribuido a la activación de este mecanismo, ya que primero se habla de la explosión y, luego, se pregunta por la diferencia de color entre ratones. Sería interesante comprobar si cambiando el diseño del problema aparecen este tipo de respuestas, por ejemplo, preguntando primero por la variación intraespecífica preexistente y, después, por las diferencias de frecuencia en la distribución de la población antes y después de la explosión.

\section{ConCLUSIONES}

La elaboración de un modelo científico escolar sobre evolución para educación primaria es un proceso complejo, pues requiere atender a distintas perspectivas y a distintos niveles de organización escalar; y, al mismo tiempo, es un instrumento idóneo para guiar el proceso de enseñanza ya desde sus primeras fases.

El modelo científico escolar propuesto recoge el contenido escolar básico señalado por los especialistas, profesorado y normativa curricular analizados. Posibilita confeccionar materiales, como la prueba inicial presentada, que respondan a la visión integrada de las distintas ideas consideradas en la construcción del modelo de evolución. Usado para analizar las respuestas de los estudiantes, ha servido para conocer cuáles de esas ideas han movilizado y en qué niveles de organización escalar. En conclusión, el modelo científico escolar propuesto resultaría útil para planificar la enseñanza, diseñar materiales y evaluar al alumnado.

Su forma de representación permitiría la sucesiva incorporación de nuevas ideas nucleares (en forma de triángulo, añadiendo lados a la figura) a medida que se progresa hacia etapas educativas superiores, facilitando así la transición de los modelos mentales del alumnado hacia modelos cada vez más completos y complejos.

En línea con la descripción de modelos como herramienta epistémica de Knuuttila (2011), este modelo, como cualquier otro, presenta potencialidades y limitaciones por su diseño (en forma de heptágono), su medio de representación (2D), su utilidad (su objetivo es guiar el proceso de construcción del modelo de evolución y evaluarlo), su carácter manipulativo (que sea manejable) y la relación que establece entre teoría y realidad (mostraría de forma sencilla las ideas nucleares necesarias en la construcción del modelo de evolución).

Hay que continuar explorando sus posibilidades y limitaciones a través de nuevos contextos e investigaciones, y comprender que, para que el profesorado de educación primaria pueda usarlo de forma efectiva, es necesario proporcionarle formación específica sobre la enseñanza y aprendizaje de la evolución. En cualquier caso, ha resultado exitoso para esta investigación porque ha permitido aproximarse al conocimiento previo de cada estudiante a través de los modelos expresados. Debemos señalar nuevamente que esto no significa conocer los modelos mentales del alumnado, ya que los modelos expresados son una representación simplificada (Franco y Colinvaux 2000).

En relación a las preconcepciones, una conclusión relevante de este análisis es la presencia de tantas, y tan variadas, ideas en torno al fenómeno evolutivo en el alumnado de educación primaria. Lo que no solo revela una exposición temprana a esta teoría, sino también la capacidad de los estudiantes para generar explicaciones razonablemente plausibles; a pesar de que no han recibido enseñanza formal al respecto, ya que en España la teoría de la evolución no es abordada en primaria. Los resultados muestran su interés por el tema y la necesidad de poder contar con modelos que expliquen cuestiones que tienen que ver con su día a día, con su curiosidad por entender el mundo que los rodea. Una necesidad a la que no se responde desde la escuela.

Además, el número de concepciones que pueden resultar un obstáculo para su aprendizaje ha resultado ser muy bajo, sobre todo en comparación con el de estudiantes de secundaria. Por el contrario, la mayoría de sus preconcepciones pueden constituir un buen punto de partida para comenzar a construir este modelo científico. 
En definitiva, cuándo y cómo enseñemos la evolución en la educación anterior a la formación universitaria es un poderoso factor para evitar su incomprensión, para fomentar las actitudes positivas de la ciudadanía hacia la teoría evolutiva, una ciudadanía que debe estar capacitada para comprender, argumentar y decidir con conocimiento científico. Las ideas escogidas para crear los modelos científicos escolares serán los ladrillos que permitirán al alumnado edificar progresivamente el modelo de evolución biológica, elegir u omitir contenidos en una etapa influirá en la comprensión de la evolución.

\section{Materiales SUPlementarios}

Anexo I (pdf)

\section{Agradecimientos}

Esta investigación ha sido financiada por el Proyecto PGC2018-096581-B-C22 subvencionado por el Ministerio de Ciencia, Innovación y Universidades y el Programa de Ayuda Predoctoral de Xunta de Galicia ED48A-2016/291. Muchas gracias al alumnado y docente participante por su entusiasmo y su colaboración.

\section{REFERENCIAS}

Adúriz-Bravo A., Izquierdo-Aymerich M. (2009) Un modelo de modelo científico para la enseñanza de las ciencias naturales. Revista Electrónica de Investigación en Educación en Ciencias 4, número especial 1, 40-49.

Alters B. J., Nelson C. E. (2002) Perspective: Teaching evolution in higher education. Evolution 56, 1891-1901.

Andersson B., Wallin A. (2006) On developing content-oriented theories taking biological evolution as an example. International Journal of Science Education 28, 673-695.

Asghar A., Wiles J. R., Alters B. (2007) Canadian pre-service elementary teachers' conceptions of biological evolution and evolution education. McGill Journal of Education 42 (2), 189-210.

Banet E., Ayuso E. G. (2003) Teaching of biological inheritance and evolution of living beings in secondary school. International Journal of Science Education 25 (3), 373-407. https://doi.org/10.1080/09500690210145716

Boulter C.J., Buckley B.C. (2000) Constructing a Typology of Models for Science Education (pp. 41-57) en Gilbert J.K., Boulter C.J. (Eds), Developing Models in Science Education. Springer: Dordrecht.

Catley K. M., Lehrer R., Reiser B. (2005) Tracing a prospective learning progression for developing understanding of evolution. Paper commissioned by the National Academies Committee on Test Design for K-12 Science Achievement.

Chamizo J. A. (2010) Una tipología de los modelos para la enseñanza de las ciencias. Revista Eureka sobre Enseñanza y Divulgación de las Ciencias 7 (1), 26-41. https://revistas.uca.es/index.php/eureka/article/view/2626/2275

Clement J. (2000) Model based learning as a key research area for science education. International Journal of Science Education 22(9), 1041-1053. https://doi.org/10.1080/095006900416901

Criado A. M., Cruz-Guzmán M., García-Carmona A., Cañal P. (2014) ¿Cómo mejorar la educación científica de primaria en España desde el currículo oficial? Sugerencias a partir de un análisis curricular comparativo en torno a las finalidades y contenidos de la ciencia escolar. Enseñanza de las Ciencias 32 (3), 249-266. https://doi.org/ $10.5565 /$ rev/ensciencias. 1069

Franco C., Colinvaux D. (2000) Grasping Mental Models (pp. 93-118) en Gilbert J.K., Boulter C.J. (Eds), Developing Models in Science Education. Springer: Dordrecht. https://doi.org/10.1007/978-94-010-0876-1_5

García Rovira M. P. (2005) Los modelos como organizadores del curriculum de biología. Enseñanza de las Ciencias, extra VII International Conference, 1-6. 


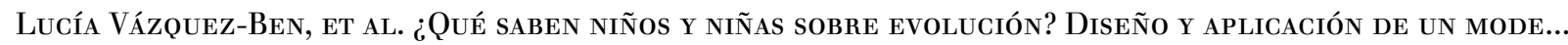

Giere R. N. (1988) Explaining science. A cognitive approach. Chicago: Chicago University Press.

Giere R. N. (1999) Using models to represent reality (pp. 41-57) en Magnani L., Nersessian N. J., Thagard P. (Eds), Model-based reasoning in scientific discovery. New York (EEUU): Kluwer and Plenum.

Giere R. N. (2004) How models are used to represent reality. Philosophy of Science 71, 742-752.

Gilbert J. K. (1993) Models \& modelling in science education. Hatfield, UK: The Association for Science Education.

Gilbert J. K. (2004) Models and Modelling: Routes to More Authentic Science Education. International Journal of Science and Mathematics Education 2, 115-130.

Gilbert J. K., Boulter C. J. (1998) Learning Science through Models and Modelling (pp. 53-56) en Fraser B. J., Tobin K. G. (Eds), International Handbook of Science Education. London: Kluwer Academic.

Gilbert J. K., Justi R. (2016) Modelling-based teaching in Science Education. Swirtzerland: Springer.

Gómez A. A., Sanmartí N., Pujol R. M. (2007) Fundamentación teórica y diseño de una unidad didáctica para la enseñanza del modelo de ser vivo en la escuela primaria. Enseñanza de las Ciencias 25(3), 325-340. https://dd d.uab.cat/record/39791

Gregory T. R. (2009) Understanding Natural Selection: essential concepts and common misconceptions. Evolution: Education and Outreach 2, 156-175. https://doi.org/10.007/s12052-009-0128-1

Ha M., Nehm R. (2014) Darwin's difficulties and students' struggles with Trait Loss: cognitive-historical parallelisms in evolutionary explanation. Science and Education 23, 1051-1074. https://doi.org/10.1007/s11191-013-962 6-1

Harms U., Reiss M. (2019) The Present Status of Evolution Education (pp. 1-19) en Harms U. y Reiss, M. J. (Eds), Evolution Education Re-considered. Swirtzerland: Springer. https://doi.org/10.1007/978-3-030-14698-6

Harrison A. G., Treagust D. F. (2000) A typology of school science models. International Journal of Science Education 22(9), 1011-1026. https://doi.org/10.1080/095006900416884

Hermann R. S. (2011) Breaking the cycle of continued evolution education controversy: on the need to strengthen elementary level teaching of evolution. Evolution: Education and Outreach 4, 267-274. https://doi.org/10.10 07/s12052-011-0325-6

Knuuttila T. (2011) Modelling and representing: an artefactual approach to model-based representation. Studies in History and Philosophy of Science 42(2), 262-272. https://doi.org/101016/j.shpsa.2010.11.034

Legare C. H., Lane J. D., Evans E. M. (2013) Anthropomorphizing Science: How does it affect the Development of Evolutionary Concepts? Merrill-Palmer Quaterly 59, 168-197. https://doi.org/10.1353/mpq2013.0009

Lehrer R., Schauble L. (2012) Seeding evolutionary thinking by engaging children in modeling its foundations. Science Education 96 (4), 701-724.

Martí J. (2012) Aprender ciencias en la educación primaria. Barcelona: Graó.

McVaugh N. K., Birchfield J., Lucero M. N., Petrosino A. J. (2011) Evolution Education: Seeing the Forest for the Trees and Focusing Our Efforts on the Teaching of Evolution. Evolution: Education and Outreach 4, 286-292. http://doi.org/10.1007/s12052-010-0297-y

Miles M. B., Huberman A. M., Saldaña J. (2013) Qualitative Data Analysis: A Methods Sourcebook. USA: SAGE Publications.

Norman D. A. (1983) Some observations on Mental Models (pp.7-13) en Gentner D., Stevens A. L. (Eds), Mental Models. Hillsdale (New Jersey, USA): LEA.

Oh P. S., Oh S. J. (2011) What teachers of Science need to know about models: An overview. International Journal of Science Education 33(8), 1109-1130. https://doi.org.10.1080/09500693.2010.502191

Oliva J. M., Aragón M. M., Bonat M., Mateo J. (2003) Un estudio sobre el papel de las analogías en la construcción del modelo cinético-molecular de la materia. Enseñanza de las Ciencias 21 (3), 429-444.

Orden ECI/3960/2007, de 19 de diciembre, por la que se establece el currículo y se regula la ordenación de la educación infantil. $B O E \mathrm{n}^{\circ} 5,5$ de enero de 2008. 
Passmore C., Svoboda J., Giere R. (2014) Models in Science and in Learning Science: Focusing Scientific Practice on Sense-making (pp. 1171-1202) en Matthews, M. R. (Ed.), International Handbook of Research in History, Philosophy and Science Teaching. Swirtzerland: Springer.

Pobiner B. (2016) Accepting, Understanding, Teaching, and Learning (Human) Evolution: Obstacles and Opportunities. American Journal of Physical Anthropology 159 (S61), 232-274. https://doi.org/10.1002/ajpa. 22910

Rodríguez Gómez G., Gil Flores J., García Jiménez E. (1996) Metodología de la investigación cualitativa. Málaga: Ediciones Aljibe.

Roldán Muñoz J. (2008) Una actividad colectiva e interactiva para comprender los conceptos más importantes de la teoría de la evolución biológica. Alambique 58, 112-119.

Simons H. (2011) El estudio de caso: teoría y práctica. Madrid: Morata

Stake R. E. (2010) Qualitative research: studying how things work. New York (USA): Guildford Press.

Vázquez-Ben L., Bugallo-Rodríguez Á. (2017) El modelo de evolución en educación primaria: desafíos identificados por expertas y expertos. Enseñanza de las ciencias Núm. Extra, 4293-4298.

Vázquez-Ben L., Bugallo-Rodríguez Á. (2018) El modelo de evolución biológica en el curriculum de Educación Primaria: Un análisis comparativo en distintos países. Revista Eureka sobre Enseñanza y Divulgación de las Ciencias 3, 3101-3113.https://doi.org/10.25267/Rev_Eureka_ensen_divulg_cienc.2018.v15.i3.3101

Vosniadou S. (2013) Conceptual Change in Learning and Instruction: The Framework Theory Approach (pp. 1130) en Vosniadou S. (Ed), International Handbook of Research on Conceptual Change (2nd Edition). New York: Routledge.

\section{INFORMACIÓN ADICIONAL}

Para citar este artículo: Vázquez-Ben L. y Bugallo-Rodríguez Á. (2022) ¿Qué saben niños y niñas sobre evolución? Diseño y aplicación de un modelo científico escolar de evolución para educación primaria. Revista Eureka sobre Enseñanza y Divulgación de las Ciencias 19(1), 1102. doi:10.25267/ Rev_Eureka_ensen_divulg_cienc.2022.v19.i1.1102 